\title{
Endocardial Injury During Ventricular Assistance with an Intracardiac Microaxial Pump
}

\author{
Angel L. Fernández ${ }^{*}, 1$, Amparo Martínez², Julian Alvarez³, José B. García-Bengochea ${ }^{1}$, \\ Jerónimo Forteza ${ }^{4}$ and José R. González-Juanatey ${ }^{2}$
}

\author{
Divisions of ${ }^{1}$ Cardiac Surgery, ${ }^{2}$ Cardiology, ${ }^{3}$ Anesthesiology and ${ }^{4}$ Pathology, University Hospital, Servizo Galego de \\ Saúde, Department of Surgery, University of Santiago de Compostela, Spain
}

\begin{abstract}
We report the case of a patient unweanable from cardiopulmonary bypass who underwent transaortic implantation of an Impella Recover LD blood pump. The ventricular assistance afforded an adequate flow but the patient died 48 hours after due to severe ischemia of the lower limbs. Chronic atherosclerotic occlusion of the abdominal aorta could be observed during the necropsy study as well as a subendocardial hematoma of the left ventricular wall. Some aspects concerning the pathologic finding observed in the heart are discussed.
\end{abstract}

\section{BACKGROUND}

The Impella Recover ${ }^{\circledR}$ LD (Impella CardioSystems, $\mathrm{GmbH}$, Aachen, Germany) is an intravascular microaxial blood pump designed for short-term left ventricular circulatory support. It has been successfully used in postcardiotomy low out-put syndrome refractory to intra-aortic balloon pump and inotropic drugs [1-3]. It consists of a straight flexible cannula which is surgically inserted through the ascending aorta across the aortic valve into the left ventricle. It can deliver up to 5.0 liters per minute of nonpulsatile flow from the left ventricle into the ascending aorta [4]. This support allows the left ventricle to rest and recover by actively unloading, reducing myocardial workload and oxygen consumption while maintaining adequate cardiac output and organ perfusion. The combination of intra-aortic balloon pump and the Impella device may provide additional hemodynamic and myocardial improvement compared to either stand-alone support [5].

We present the case of patient who died after Impella Recover $^{(B)}$ LV support for postcardiotomy shock. Some aspects concerning pathological findings suppress are discussed.

\section{CASE PRESENTATION}

A 66-year old male with past history of chronic obstructive airway disease, tobacco consumption, transient ischemic attack and chronic lower limb ischemia was admitted to our Emergency Department because of acute onset of chest pain. The ECG revealed a sinus tachycardia at 123 beats per minute and a ST segment elevation suggestive of acute anterolateral myocardial infarction. Immediately after admission the patient developed dyspnea, respiratory failure and severe hypotension. Orotracheal intubation, mechanical ventilation and inotropic support were required.

*Address correspondence to this author at the Division of Cardiac Surgery, University Hospital, Department of Surgery, University of Santiago de Compostela, Ave. Choupana, s/n. 15706 Santiago de Compostela, Spain; Tel: +34-981-950-212; Fax: +34-981-950-227;

E-mail: alfg@secardiologia.es
The patient was urgently transferred to the catheterization laboratory. Transradial coronary angiogram demonstrated chronic occlusion of the right coronary, severe stenosis of the left main coronary artery, as well as, severe stenosis of the proximal left anterior descending and circumflex arteries. The distal right coronary artery was not graftable and received flow from the left anterior descending artery.

Transfemoral insertion of an intra-aortic balloon pump was not possible due to femoro-ilicac occlusion. A transthoracic echocardiogram revealed severe depression of the left ventricular systolic function with moderate mitral incompetence. Arterial hypotension, low cardiac output, oliguria and severe cyanosis of the limbs persisted in spite of maximum inotropic support. The patient was referred to our Cardiac Surgical Unit for emergency coronary artery bypass grafting.

The patient suffered an episode of ventricular fibrillation after opening the sternum which forced to an emergency instauration of cardiopulmonary bypass. Arterial grafts were not considered and the left great saphenous vein was harvested. Two saphenous vein coronary artery bypass grafts were implanted in the left anterior descending and marginal coronary arteries using standard cardiopulmonary bypass with aortic crossclamping. Cold blood cardioplegia was given through the aortic root and the coronary sinus. Weaning from cardiopulmonary bypass was not possible despite of excellent flow through the grafts and maximum inotropic support. An intraopertive transesophageal echocardiogram evidenced severe and global depression of the left ventricular function with a left ventricular ejection fraction lower than $15 \%$ and moderate mitral incompetence. Mitral disease was assumed to be a consequence of acute dilation of the left ventricle.

An Impella Recover ${ }^{\circledR}$ LD was inserted through a $10 \mathrm{~mm}$ Dacron graft sutured end-to-side to the ascending aorta. The microaxial pump was advanced across the aortic valve and placed in the left ventricle under hemodynamic and ultrasound monitoring (Fig. 1). The Impella Recover ${ }^{\mathbb{B}}$ LD assist started and was set to maximum support obtaining blood pump of $5.1 \mathrm{l} / \mathrm{min}$. Effective left ventricular unloading al- 


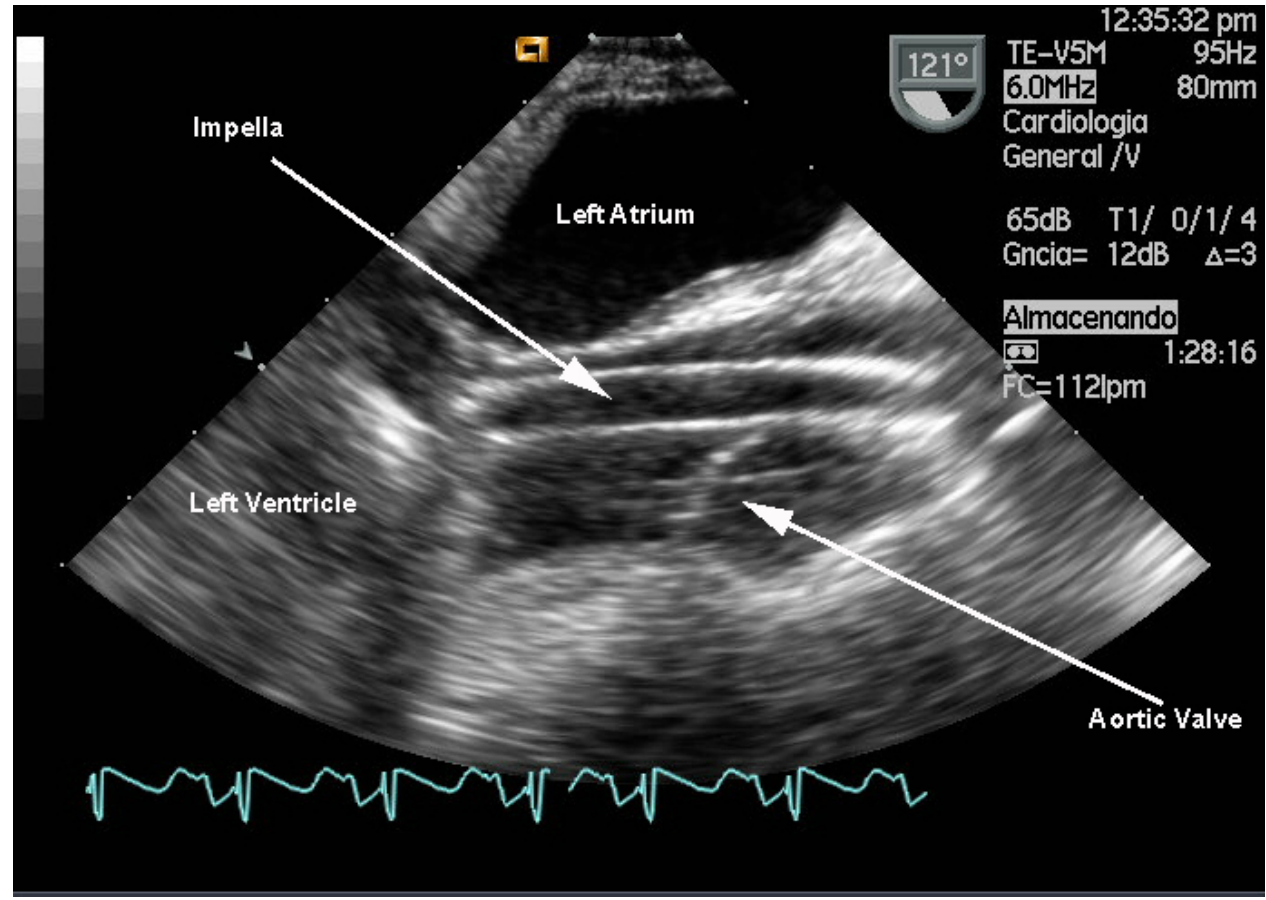

Fig. (1). Two dimensional transesophageal echocardiography. The tip of pump is placed in the left ventricle outflow tract across the aortic valve.

lowing easy and quick cardiopulmonary bypass weaning maintaining a mean arterial pressure of $60-65 \mathrm{mmHg}$. Once cardiopulmonary bypass was discontinued, aortic and atrial cannulas were removed, protamine was given and the sternum closed as usually while the shaft catheter of the Impella came out of the thorax through a small skin incision over the incisura jugularis of the sternum.
The patient was transferred to the postoperative care unit. The Impella Recover ${ }^{\circledR}$ LD was able to work at the highest performance without problems. Postoperative chest X-ray confirmed the position of the Impella (Fig. 2). The cardiac output tested by the pulmonary artery catheter was maintained around 5.2 to 5.6 liters per minute with a median arterial pressure of $60-65 \mathrm{mmHg}$ without inotropic support. Intravenous sodium heparin was given to maintain an activated

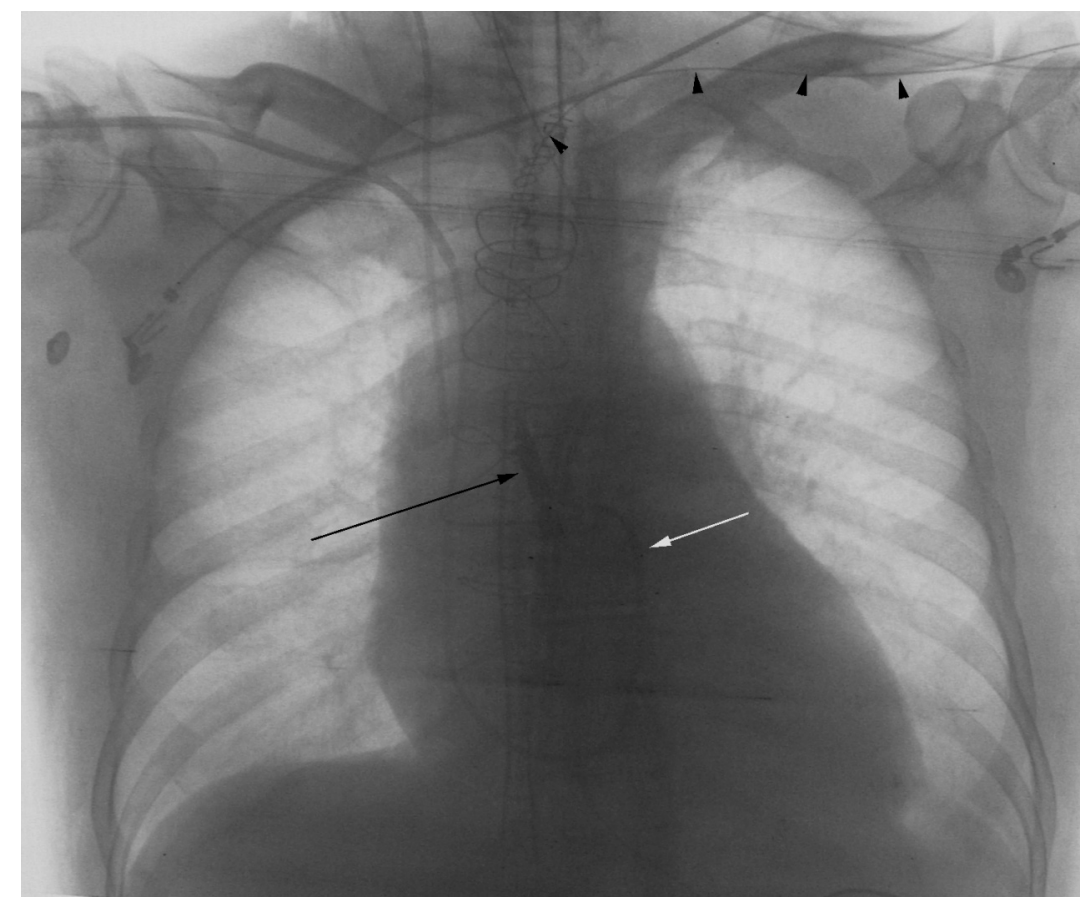

Fig. (2). Postoperative chest-X-ray film. The microaxial pump is implanted in the left ventricle through the ascending aorta (black arrow). The shaft catheter comes out of the thorax through the incisura jugularis of the sternum (black arrowheads). The Swan-Ganz catheter is placed in the pulmonary artery (white arrow). 
coagulation time between 130 and 150 seconds. A small pulse wave was observed as well as an adequate oxygenation, low capillary wedge pressure and more than $1 \mathrm{ml} / \mathrm{kg}$ of spontaneous diuresis. However signs of severe lower limb ischemia persisted.

After 24 hours of appropriate support a firs attempt of weaning from the Impella Recover ${ }^{\circledR}$ was made by reducing the support. A transesophageal echocardiogram showed slight recovery of ventricular systolic function with the pump set in medium support but insufficient for a complete weaning.

48 hours after the ventricular support started, signs of severe ischemia and necrosis of the lower limbs were evident and associated to raised serum concentration of creatine kinase and myoglobin. Surgical or percutaneous revascularization of the lower limbs was ruled out and after informed consent the blood pump was stopped and the patient died six hours later. The necropsy study demonstrated absence of any injury of the aortic valve. However a subendocardial hematoma of the muscular aspect of the left ventricular outflow tract could be observed (Fig. 3) as well as a chronic atherosclerotic occlusion of the distal abdominal aorta.

\section{COMMENTS}

The Impella Recover ${ }^{\circledR}$ LD is easy to implant and efficiently supports the heart in post-cardiotomy shock patients ranging assist time between 7 and 228 hours. Improved survival has been observed in cases with residual cardiac func- tion higher than $1 \mathrm{~L} / \mathrm{min}$ [1]. An adequate mean arterial pressure and cardiac output in absence of a peak of systolic pressure may guarantee peripheral organ perfusion unless a severe arterial stenosis is present as occurred in the case reported. However we do not believe that preoperative lower limb ischemia in a patient with cardiogenic chock constitutes a contraindication for continuous flow-pump circulatory support. Perhaps a more aggressive approach to postoperative limb ischemia -for example an extraanatomic axilobifemoral bypass- should had been taken into account in our case.

The soft tip and the surface characteristics of the cannula may contribute to avoid aortic valve and endocardial injury during Impella Recover ${ }^{\mathbb{B}}$ LD implantation and ventricular unloading support. Neither aortic valve injuries nor device dysfunction or thromboembolic complications have been described. However it is possible that contraction and unloading of the heart may displace the device allowing the intraventricular shaft to tap the wall leading to slight endocardial suction and hematoma.

Ecocardiogram-Doppler examinations during Impella Recover ${ }^{B}$ LD support have demonstrated absence of pump induced aortic regurgitation even in patients with bioprosthetic aortic valves and pulmonary autografts [6, 7]. However cases of functional mitral stenosis secondary to pump placement have been reported [8]. Minimal blood damage during pumping and a light anticoagulation level required provide a low rate of hemorrhagic complications [1-4].

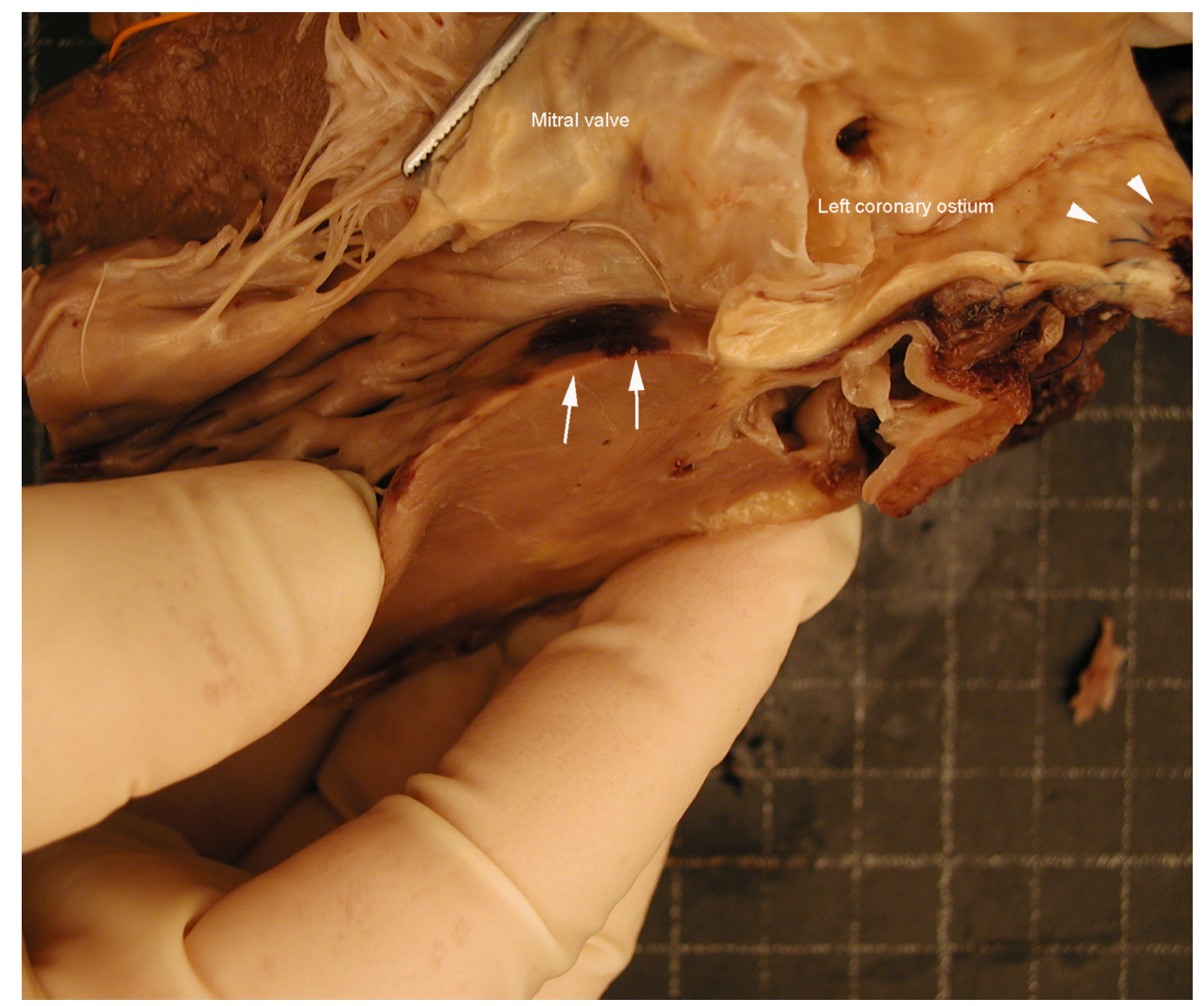

Fig. (3). Macroscopic image of the heart obtained during the necropsy study. A subendocardial hematoma is observed on the muscular aspect of the left ventricle outflow tract (white arrows). The proximal anastomosis of a saphenous graft is observed (white arrowheads). 


\section{REFERENCES}

[1] Siegenthaler MP, Brehm K, Strecker T, et al. The impella recover microaxial left ventricular assist device reduces mortality for postcadiotomy failure: a three- center experience. J Thorac Cardiovasc Surg 2004; 127: 821-2.

[2] Jurmann MJ, Siniawski H, Erb M, Drews T, Hetzer R. Initial experience with miniature axial flow ventricular assist devices for postcardiotomy heart failure. Ann Thorac Surg 2004; 77: 1642-7.

[3] Onorati F, Cristodoro L, Borrello F, Esposito A, Merola S, Renzulli A. Ventricular assistance with microaxial flow pump following mitral repair for dilated cardiomyopathy. Int J Artif Organs 2006; 29: $591-7$.

[4] Bautista-Hernández V, Gutiérrez F, Pinar E, et al. Initial experience with the Impela left ventricular assist device for postcardiotomy cardiogenic shock and unprotected left coronary artery an- gioplasty in patients with a low left ventricular ejection fraction. Rev Esp Cardiol 2007; 60: 984-7.

[5] Sauren LD, Accord RE, Hamzeh K, et al. Combined Impella and intra-aortic balloon pump support to improve both ventricular unloading and coronary blood flow for myocardial recovery: an experimental study. Artif Organs 2007; 31: 839-42.

[6] Lauten A, Franke U, Strauch JT, Kaluza M, Wahlers T. Postcardiotomy failure after Ross operation: implantation of intravscular flow pump through pulmonary autograft. Thorac Cardiovasc Surg 2007; 55: 399-400.

[7] Strauch JT, Franke UF, Breuer M, et al. Technically feasibility of impella recover 100 microaxial left ventricular assit device placement after biologic aoritc valve repalcement $(21 \mathrm{~mm})$ for postcardiotomy failure. J Thorac Cardiovasc Surg 2005; 130: 1715-6.

[8] Toggweiler S, Jamshidi P, Erne P. Functional mitral stenosis: a rare complication of the Impella assist device. Eur J Echocardiogr 2008; 9: 412-3.

(C) Fernández et al.; Licensee Bentham Open.

This is an open access article licensed under the terms of the Creative Commons Attribution Non-Commercial License (http: //creativecommons.org/licenses/by-nc/ $3.0 /$ ) which permits unrestricted, non-commercial use, distribution and reproduction in any medium, provided the work is properly cited. 